\title{
Endoscopic Drainage of Orbital Subperiosteal Hematoma Secondary to Acute Rhinosinusitis in a Child
}

\author{
Kazuhiro Nomura, ${ }^{1}$ Yohei Honkura, ${ }^{1}$ Daiki Ozawa, ${ }^{1}$ Yuri Okumura, ${ }^{1}$ \\ Hiroshi Hidaka, ${ }^{1}$ Takenori Ogawa, ${ }^{1}$ Daisuke Yamauchi, ${ }^{1}$ Kenichi Watanabe ${ }^{1}$ \\ and Yukio Katori ${ }^{1}$
}

${ }^{1}$ Department of Otolaryngology-Head and Neck Surgery, Tohoku University Graduate School of Medicine, Sendai, Miyagi, Japan

\begin{abstract}
Acute rhinosinusitis is frequently associated with secondary orbital infection, most commonly subperiosteal abscess. Although orbital subperiosteal abscess is a deadly disease that might lead to blind and cavernous sinus thrombosis, recent review of literature showed that immediate surgical intervention might not always be necessary for subperiosteal abscess. Orbital inflammation secondary to sinusitis is common in children, whereas orbital subperiosteal hematoma secondary to sinusitis is extremely rare, with only 11 reported cases, including one case in children. All the cases were treated with surgical intervention. Here we present a 12-year-old girl with rhinosinusitis and proptosis. Emergent endoscopic sinus surgery with partial removal of the lamina papyracea revealed dark brown fluid in the subperiosteal space. The patient was symptom-free 2 weeks after surgery. The present case was treated exclusively via an endonasal approach, whereas all 11 previous cases of subperiosteal hematoma were treated with external incision. The endonasal approach is favorable, especially for young female patients. Our review of literature shows that sudden onset, afebrile, and few signs of inflammation on blood test in patients with subperiosteal lesion may indicate subperiosteal hematoma. Surgery rather than antibiotic administration should be considered for the treatment of suspected subperiosteal hematoma. Treatment through only the endonasal approach is possible even if the hematoma is located in the roof of the orbit.
\end{abstract}

Keywords: hematoma; infection; orbital subperiosteal abscess; sinusitis; surgery

Tohoku J. Exp. Med., 2014 July, 233 (3), 171-174. C 2014 Tohoku University Medical Press

\section{Introduction}

Acute rhinosinusitis is more common in children than in adults (Chandler et al. 1970; Eviatar et al. 2008), with the most affected age between 3 and 6 years old (Sultész et al. 2009). Acute bacterial rhinosinusitis is a common complication of viral upper respiratory infection or allergic inflammation. Using stringent criteria to define acute sinusitis, it has been observed that be- tween $6 \%$ and $7 \%$ of children seeking care for respiratory symptoms has an illness consistent with this definition (Wald et al. 2013). Acute rhinosinusitis is frequently associated with secondary orbital infection, most commonly subperiosteal abscess in children (Reid 2004). Subperiosteal abscess and orbital abscess are severe types of orbital infection. Conventionally, emergency endoscopic sinus surgery combined with intravenous antibiotics is the first choice for the treatment of suspected abscess (Eviatar et al. 2008; Sultész et al. 2009; Kinis et al. 2013). However, immediate surgical intervention may not always be necessary for subperiosteal abscess (Bedwell and
Bauman 2011; Bedwell and Choi 2013). Orbital subperiosteal hematoma is rare and occurs most often after trauma (Dobben et al. 1998). Subperiosteal hematoma secondary to sinusitis is extremely rare with only 11 reported cases (Wheeler 1937; Harris et al. 1978; Leopold et al. 1980; Ichino et al. 1985; Choi et al. 1988; Zalzal 1991; Aoki et al. 1997; Woo and Kim 1997; Park et al. 2010). Only one of these 11 cases was aged under 18 years (Zalzal 1991). All 11 previous patients with subperiosteal hematoma were treated with surgery. Here we describe our treatment of a 12-year-old girl with acute rhinosinusitis and subperiosteal hematoma, and discuss the difference between subperiosteal hematoma and abscess.

\section{Case Report}

A 12-year-old girl with no history of immunodeficiency or congenital disorder presented to the emergency department with a 3-day history of swollen, erythematous, painful left eye and nasal obstruction. She had had a common cold for one week. Three days previously she had

Received May 12, 2014; revised and accepted June 4, 2014. Published online June 26, 2014; doi: 10.1620/tjem.233.171.

Correspondence: Kazuhiro Nomura, M.D., Department of Otolaryngology-Head and Neck Surgery, Tohoku University Graduate School of Medicine, 1-1 Seiryo-machi, Aoba-ku, Sendai, Miyagi 980-8574, Japan.

e-mail: kazuhiroe@gmail.com 


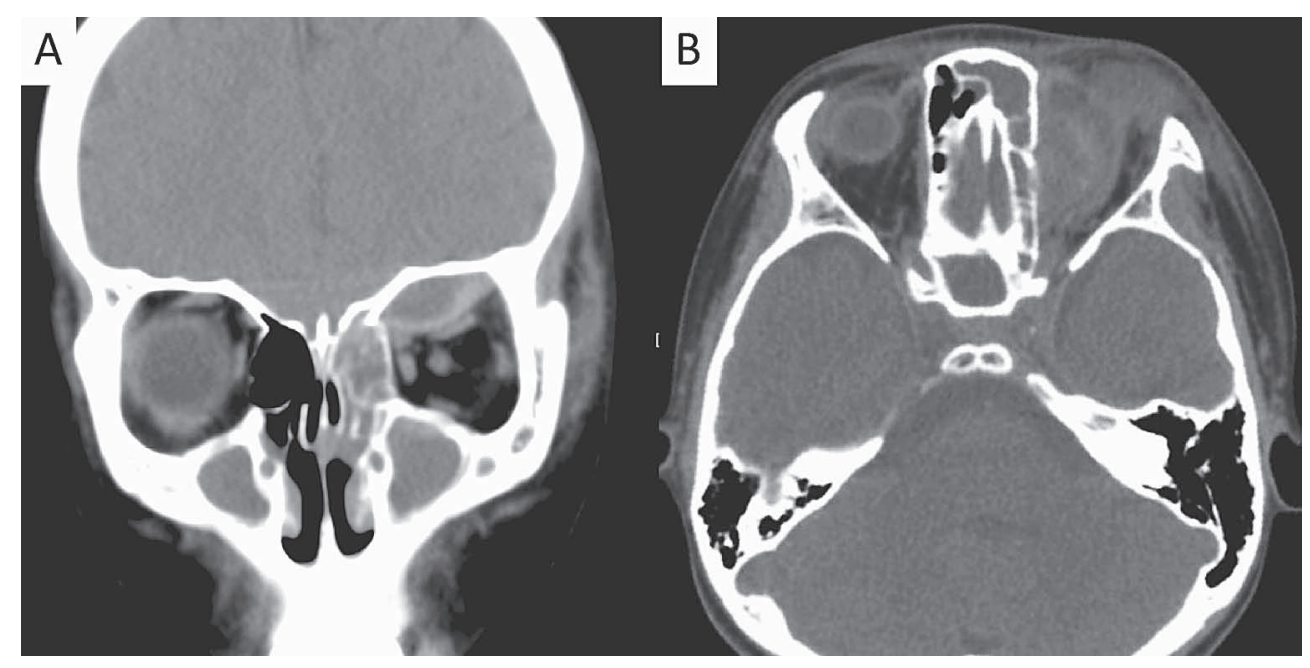

Fig. 1. Preoperative computed tomography scans.

(A) Coronal scan. Both maxillary sinuses and the left ethmoid sinus are opacified. Lens-shaped lesion is seen in the left orbit. Thickened orbital periosteum is seen.

(B) Axial scan. Left frontal and ethmoid sinuses are opacified. The lesion in the left orbit has displaced the upper rectus muscle.

headache early in the morning and noticed that her left eye was swelling when she woke up. She visited an ophthalmology clinic. She was diagnosed with chalazia and topical antibiotic was prescribed. However, the swelling worsened. When she visited the same clinic 3 days later, orbital subperiosteal abscess was suspected and she was referred to our department.

Physical examination found swollen left upper and lower eyelid without discharge. Eye movement was severely restricted and diplopia was present in all directions. Vision was 1.0 and fundoscopy found no abnormalities. Intraocular pressure was $14 \mathrm{mmHg}$ (normal range $10-20 \mathrm{mmHg}$ ). Mild proptosis was observed with a Hertel exophthalmometer $(8 \mathrm{~mm}$ on the right and $13 \mathrm{~mm}$ on the left). Pupillary light reflex was normal. She was afebrile. Complete blood cell count showed no abnormality, with a white blood cell count of $8,400 / \mathrm{mm}^{3}$ and C-reactive protein (CRP) level of $15 \mathrm{mg} / \mathrm{L}$. Computed tomography (CT) revealed bilateral maxillary and left fronto-ethmoidal sinusitis, and a subperiosteal lesion in the roof of the left orbit (Fig. 1).

Endoscopic sinus surgery was performed under general anesthesia. The lateral nasal wall was extremely edematous (Fig. 2A and B). Mucosa of the sinuses was also edematous. No purulent discharge was observed. After removing the mucosa of the lamina papyracea, dark brown fluid was seen through the papyracea (Fig. 2C). After dissection between the papyracea and orbital periosteum, dark blood and clot were drained (Fig. 2D). After irrigation with saline, the nasal cavity was packed loosely with chitincoated gauze. Ampicillin sodium $1 \mathrm{~g}$ and sulbactam sodium $0.5 \mathrm{~g}$ were intravenously administered every 6 hours for 4 days. Later, oral clarithromycin was administered. The packed gauze was removed 2 days after surgery. The proptosis slowly resolved and all symptoms had disappeared in
2 weeks.

\section{Discussion}

Sinusitis is the primary cause of orbital complications, accounting for $74 \%$ to $85 \%$ of cases (Kinis et al. 2013). Orbital complications secondary to sinusitis are mostly orbital infections and can be staged according to Chandler's classification (Chandler et al. 1970) into five groups (Group I: inflammatory edema, Group II: orbital cellulitis, Group III: subperiosteal abscess, Group IV: orbital abscess, Group V: cavernous sinus thrombosis). Subperiosteal abscess is the most common intraorbital complication in the pediatric population (Reid 2004). In children, the periosteum at the medial orbital wall is loosely attached to the underlying lamina papyracea (Reid 2004). A recent review of cases of pediatric subperiosteal abscess suggested that immediate surgical intervention is not always the best treatment option (Bedwell and Bauman 2011; Bedwell and Choi 2013). Patients presenting with advanced ophthalmologic findings (impaired visual acuity, elevated intraocular pressure, ophthalmoplegia, proptosis $\geq 5 \mathrm{~mm}$ ) or with large abscesses (width $>10 \mathrm{~mm}$ ) are best treated surgically. Patients with milder symptoms may improve with conservative management (intravenous antibiotics and topical treatment).

In our patient with subperiosteal hematoma, the CT findings were similar to those of subperiosteal abscess. Proptosis of $5 \mathrm{~mm}$ and large subperiosteal lesion were indications for immediate surgery. All 11 previous cases of subperiosteal hematoma secondary to sinusitis were treated surgically (Wheeler 1937; Harris et al. 1978; Leopold et al. 1980; Ichino et al. 1985; Choi et al. 1988; Zalzal 1991; Aoki et al. 1997; Woo and Kim 1997; Park et al. 2010). The pathogenesis of subperiosteal hematoma is thought to be rupture of vessels as a result of phlebitis in the sinus mucosa. Vessel rupture may occur after congestion or ero- 


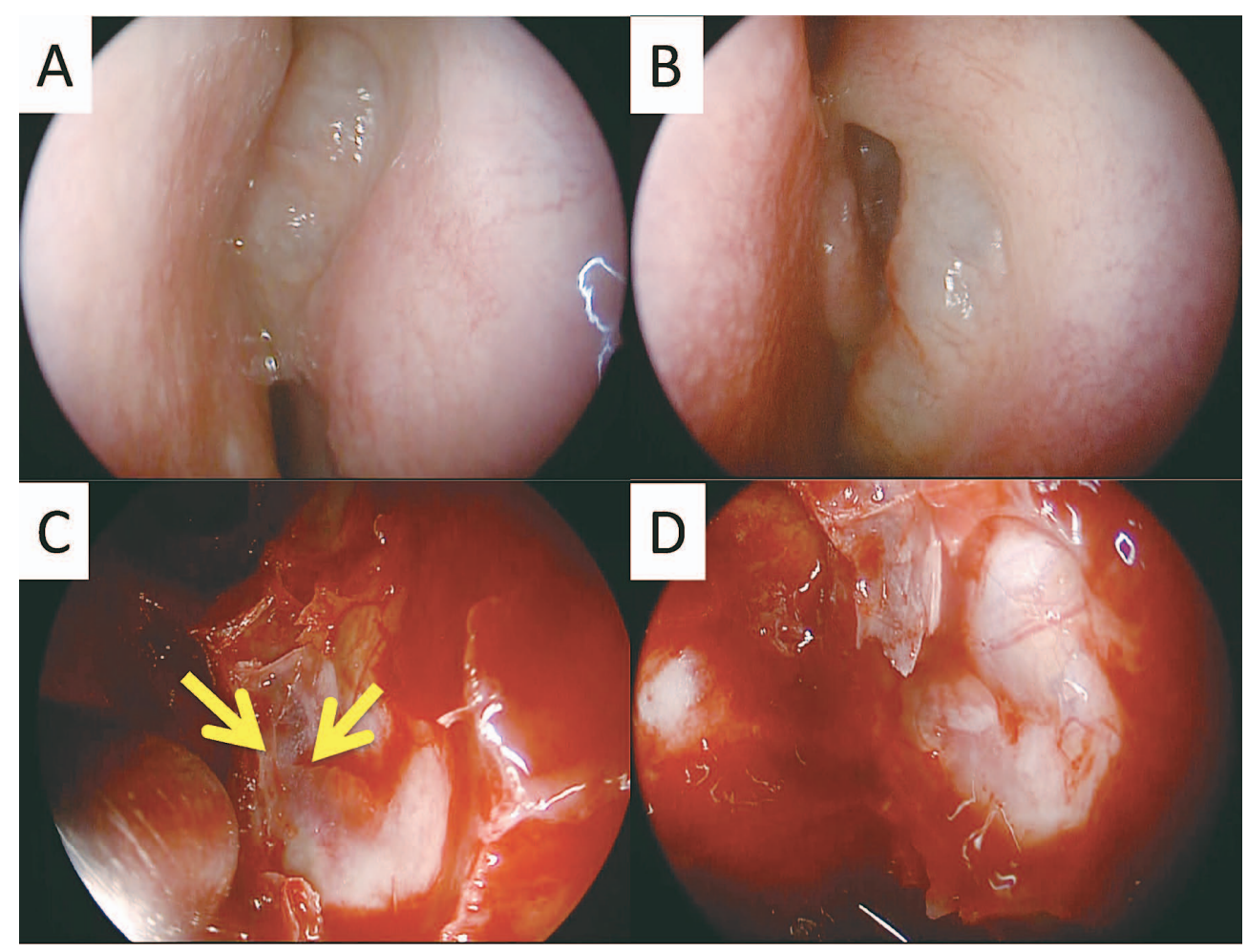

Fig. 2. Intraoperative endoscopic views.

(A) Lateral nasal wall is severely edematous.

(B) After treatment with local infiltration of adrenaline. Middle meatus is seen between the middle turbinate and still edematous lateral nasal wall.

(C) After removal of mucosa on the lamina papyracea, dark brown fluid (arrows) is seen behind the papyracea.

(D) Dark brown fluid and blood clot are drained after dissection between the lamina papyracea and subperiosteum.

Table 1. Reported cases of orbital periosteal hematoma secondary to sinusitis.

\begin{tabular}{|c|c|c|c|c|c|c|c|c|}
\hline Reference & $\begin{array}{c}\text { Age } \\
\text { (years) }\end{array}$ & Sex & Onset & Fever & $\begin{array}{l}\text { WBC } \\
\left(/ \mathrm{mm}^{3}\right)\end{array}$ & ESR or CRP & Surgery & Visual outcome \\
\hline (Wheeler 1937) & 45 & M & Gradual & na & na & na & External & Diplopia \\
\hline (Harris et al. 1978) & 30 & $\mathrm{~F}$ & Sudden & $\mathrm{N}$ & 10,600 & na & External & Full recovery \\
\hline (Leopold et al. 1980) & 56 & $\mathrm{~F}$ & Sudden & $\mathrm{N}$ & 7,000 & na & External & Full recovery \\
\hline (Ichino et al. 1985) & 57 & $\mathrm{~F}$ & Sudden & na & na & na & External & Full recovery \\
\hline (Choi et al. 1988) & 62 & $\mathrm{M}$ & Sudden & $\mathrm{N}$ & 13,600 & na & External & Full recovery \\
\hline (Zalzal 1991) & 4 & $\mathrm{~F}$ & Sudden & $\mathrm{N}$ & WNL & na & External + ESS & Full recovery \\
\hline (Woo \& Kim 1997) & 44 & $\mathrm{~F}$ & Sudden & $\mathrm{Y}$ & na & na & External + ESS & Full recovery \\
\hline (Woo \& Kim 1997) & 42 & M & na & na & na & na & External + ESS & Full recovery \\
\hline (Aoki et al. 1997) & 68 & $\mathrm{~F}$ & Sudden & $\mathrm{N}$ & WNL & WNL & External + craniotomy & Full recovery \\
\hline (Park et al. 2010) & 57 & $\mathrm{~F}$ & Sudden & $\mathrm{N}$ & 5,900 & WNL & External + ESS & Full recovery \\
\hline (Park et al. 2010) & 62 & $\mathrm{~F}$ & Gradual & $\mathrm{N}$ & 5,400 & WNL & External + ESS & Full recovery \\
\hline (Present case) & 12 & $\mathrm{~F}$ & Sudden & $\mathrm{N}$ & 8,400 & CRP $15 \mathrm{mg} / \mathrm{L}$ & ESS & Full recovery \\
\hline
\end{tabular}

M, male; F, female; WBC, white blood cell count; ESR, erythrocyte sedimentation rate; CRP, C-reactive protein; ESS, endoscopic sinus surgery; na, not available; WNL, within normal limit; N, no; Y, yes.

sion of the vessel caused by the infection (Harris et al. 1978; Choi et al. 1988). The etiology of orbital hematoma has been classified into three groups: 1) trauma (most common), 2) vascular malformations and tumors, and 3) spontaneous (rare, thought to be due to sudden increase in intraorbital pressure or systemic disease) (Skull and Sullivan
1997; Martinez Devesa 2002). Hematoma secondary to acute rhinosinusitis is rare and was not discussed in these reports.

Differentiation between subperiosteal abscess and hematoma is important, because surgery is needed for hematoma but not always for abscess. Onset of the ocular 
symptom is sudden in most patients with hematoma (Table 1). Only one of the 11 previous patients was febrile, although half of the patients had a history of upper respiratory infection (Park et al. 2010) (Table 1). The body temperature of patients with orbital subperiosteal abscess was $38.1 \pm 0.1^{\circ} \mathrm{C}$ (Kinis et al. 2013). White blood cell count varied but did not reach the extremely high levels in patients with abscess (mean \pm S.D. $17,000 \pm 1,600 / \mathrm{mm}^{3}$ ) (Kinis et al. 2013). Markers for inflammation, such as erythrocyte sedimentation rate and CRP level, were low in patients with subperiosteal hematoma (Table 1). All previous patients were treated with surgery. Since the hematoma was the cause of the orbital symptoms and most patients were afebrile, if hematoma is suspected, operative intervention rather than antibiotic administration should be considered. The outcome was favorable in all patients except the first reported in 1937 (Wheeler 1937).

All patients with subperiosteal hematoma were treated with external incision with or without the endoscopic endonasal approach (Table 1). Treatment exclusively via the endonasal approach has become possible with the development of endoscopic sinus surgery techniques and instruments. In our case, the hematoma was identified at the superomedial part of the orbit by CT. After complete fronto-ethmoidectomy and removal of the mucosa of the lamina papyracea, the dark brown hematoma could be seen through the papyracea bone. Partial removal of the papyracea and gentle dissection between the bone and periosteum allowed access to the hematoma and drainage. If the patient is young and female, as in our case, external incision should be avoided for esthetic reasons.

Subperiosteal abscess is more common in children than in adults (Chandler et al. 1970), whereas subperiosteal hematoma is more common in adults, occurred in only two children including our patient, possibly because of differences in the strength of vessels, thickness and porousness of the papyracea, and adhesion at the subperiosteal space between children and adults. Sudden onset, afebrile, and few signs of inflammation on blood test in patients with subperiosteal lesion may indicate subperiosteal hematoma. Surgery rather than antibiotic administration should be considered for the treatment of suspected subperiosteal hematoma. Treatment through only the endonasal approach is possible even if the hematoma is located in the roof of the orbit.

\section{Conflict of Interest}

The authors declare no conflict of interest.

\section{References}

Aoki, H., Tanaka, Y., Niki, Y., Kamada, K. \& Fujita, T. (1997)
Intraorbital subperiosteal hematoma due to paranasal mucocele: case report. Neurol. Med. Chir. (Tokyo), 37, 627-629.

Bedwell, J. \& Bauman, N.M. (2011) Management of pediatric orbital cellulitis and abscess. Curr. Opin. Otolaryngol. Head Neck Surg., 19, 467-473.

Bedwell, J.R. \& Choi, S.S. (2013) Medical versus surgical management of pediatric orbital subperiosteal abscesses. Laryngoscope, 123, 2337-2338.

Chandler, J.R., Langenbrunner, D.J. \& Stevens, E.R. (1970) The pathogenesis of orbital complications in acute sinusitis. Laryngoscope, 80, 1414-1428.

Choi, S., Lawson, W. \& Urken, M.L. (1988) Subperiosteal orbital hematoma. An unusual complication of sinusitis. Arch. Otolaryngol. Head Neck Surg., 114, 1464-1466.

Dobben, G.D., Philip, B., Mafee, M.F., Choi, K., Belmont, H. \& Dorodi, S. (1998) Orbital subperiosteal hematoma, cholesterol granuloma, and infection. Evaluation with MR imaging and CT. Radiol. Clin. North Am., 36, 1185-1200.

Eviatar, E., Gavriel, H., Pitaro, K., Vaiman, M., Goldman, M. \& Kessler, A. (2008) Conservative treatment in rhinosinusitis orbital complications in children aged 2 years and younger. Rhinology, 46, 334-337.

Harris, G.J., Kay, M.C. \& Nilles, J.J. (1978) Orbital hematoma secondary to frontal sinusitis. Ophthalmology, 85, 1229-1234.

Ichino, Y., Nagata, M. \& Ishikawa, T. (1985) Subperiosteal orbital hemorrhage associated with chronic sinusitis: a case report and review of the literature. Auris Nasus Larynx, 12, 27-30.

Kinis, V., Ozbay, M., Bakir, S., Yorgancilar, E., Gun, R., Akdag, M., Sahin, M. \& Topcu, I. (2013) Management of orbital complications of sinusitis in pediatric patients. J. Craniofac. Surg., 24, 1706-1710.

Leopold, D.A., Kellman, R.M. \& Gould, L.V. (1980) Retro-orbital hematoma and proptosis associated with chronic sinus disease. Arch. Otolaryngol., 106, 442-443.

Martinez Devesa, P. (2002) Spontaneous orbital haematoma. J. Laryngol. Otol., 116, 960-961.

Park, H.W., Lee, B.J. \& Chung, Y.S. (2010) Orbital subperiosteal hematoma associated with sinus infection. Rhinology, 48, 117-122.

Reid, J.R. (2004) Complications of pediatric paranasal sinusitis. Pediatr. Radiol., 34, 933-942.

Skull, S.A. \& Sullivan, T.J. (1997) Subperiosteal orbital haematoma presenting as proptosis at birth. J. Paediatr. Child Health, 33, 256-258.

Sultész, M., Csákányi, Z., Majoros, T., Farkas, Z. \& Katona, G. (2009) Acute bacterial rhinosinusitis and its complications in our pediatric otolaryngological department between 1997 and 2006. Int. J. Pediatr. Otorhinolaryngol., 73, 1507-1512.

Wald, E.R., Applegate, K.E., Bordley, C., Darrow, D.H., Glode, M.P., Marcy, S.M., Nelson, C.E., Rosenfeld, R.M., Shaikh, N., Smith, M.J., Williams, P.V. \& Weinberg, S.T. (2013) Clinical practice guideline for the diagnosis and management of acute bacterial sinusitis in children aged 1 to 18 years. Pediatrics, 132, e262-280.

Wheeler, J.M. (1937) Orbital cyst without epithelial lining: report of two cases of blood cyst. AMA Arch. Ophthalmol., 18, 356-362.

Woo, K.I. \& Kim, Y.D. (1997) Subperiosteal hematoma of the orbit associated with sinusitis. Korean J. Ophthalmol., 11, $118-122$.

Zalzal, G.H. (1991) Periorbital hematoma secondary to sinusitis in a child. Arch. Otolaryngol. Head Neck Surg., 117, 557-559. 